\title{
Pilot study of population-based newborn screening for spinal muscular atrophy in New York state
}

\author{
Jennifer N. Kraszewski, MPH ${ }^{1,2}$, Denise M. Kay, $\mathrm{PhD}^{3}$, Colleen F. Stevens, $\mathrm{PhD}^{3}$, Carrie Koval, MS ${ }^{1}$, \\ Bianca Haser, BS ${ }^{1}$, Veronica Ortiz, $\mathrm{MHS}^{1}$, Anthony Albertorio, BA ${ }^{1}$, Lilian L. Cohen, MD, MPH ${ }^{4}$, \\ Ritu Jain, PhD ${ }^{3}$, Sarah P. Andrew, BA ${ }^{5}$, Sally Dunaway Young, PT, DPT ${ }^{5}$, Nicole M. LaMarca,6, \\ Darryl C. De Vivo, MD ${ }^{1,5}$, Michele Caggana, SCD, FACMG ${ }^{3}$ and Wendy K. Chung, MD, PhD ${ }^{1,7^{\prime}}$
}

\begin{abstract}
Purpose: To determine feasibility and utility of newborn screening for spinal muscular atrophy (SMA) in New York State.

Methods: We validated a multiplex TaqMan real-time quantitative polymerase chain reaction assay using dried blood spots for SMA. From January 2016 to January 2017, we offered, consented, and screened 3,826 newborns at three hospitals in New York City and tested newborns for the deletion in exon 7 of SMN1.
\end{abstract}

Results: Ninety-three percent of parents opted in for SMA screening. Overall the SMA carrier frequency was $1.5 \%$. We identified one newborn with a homozygous SMN1 deletion and two copies of SMN2, which strongly suggests the severe type 1 SMA phenotype. The infant was enrolled in the NURTURE clinical trial and was first treated with Spinraza at age 15 days. She is now age 12 months, meeting all developmental milestones, and free of any respiratory issues.

Conclusion: Our pilot study demonstrates the feasibility of population-based screening, the acceptance by families, and the benefit of newborn screening for SMA. We suggest that SMA be considered for addition to the national recommended uniform screening panel.

Genet Med advance online publication 12 October 2017

Key Words: newborn screening; nusinersen; spinal muscular atrophy

\section{INTRODUCTION}

Spinal muscular atrophy (SMA) is the most common genetic cause of death in children under age two years ${ }^{1}$ that is autosomal recessively inherited with an incidence of approximately 1 in 5,000 to 1 in 11,000 and a carrier frequency of 1 in 54 in the United States. ${ }^{2}$ Muscle weakness in SMA is progressive, varies in severity within clinical subtypes, and includes bilateral muscle atrophy due to anterior horn cell loss. ${ }^{3}$ SMN1 exon 7 deletions account for approximately 95$98 \%$ of SMA mutations across race/ethnic groups. The SMA phenotype is variable (types $0,1,2,3$, and 4 ) and ranges in severity and onset (infancy to adult), which is modified by the number of SMN2 gene copies. ${ }^{4}$ SMN1 and SMN2 differ by five nucleotides, including an enhancer splice element that leads to exclusion of exon 7 in most SMN2 transcripts leading to an unstable, rapidly degraded protein., 5

Given the high frequency of SMA across all races/ethnicities caused by a single common mutation in $>95 \%$ of individuals, population-based screening for SMA is theoretically feasible. In addition, given the progressive nature of the motor neuron loss, early intervention to prevent motor neuron loss, even before the onset of symptoms, should allow for maximal benefit to individuals who will develop SMA. For these reasons, SMA was nominated to the Health and Human Services Secretary's Advisory Committee on Heritable Disorders in Newborns and Children for addition to the recommended uniform newborn screening panel in 2008, but was denied because of a lack of available treatment and newborn screening pilot studies demonstrating feasibility. However, the Food and Drug Administration recently gave the broadest approval for the first and only effective treatment for SMA, Spinraza (nusinersen), in December 2016. Here we describe a pilot study demonstrating the acceptance by parents and feasibility of newborn screening for SMA in New York State (NYS) and report the outcome of the first baby identified through newborn screening who was successfully treated with Spinraza.

\section{Recruitment \\ MATERIALS AND METHODS \\ All studies were approved by the institutional review boards of Columbia University, Weill Cornell Medical College, and the NYS Department of Health. Study coordinators approached English- and Spanish-speaking postpartum}

\footnotetext{
${ }^{1}$ Department of Pediatrics, Columbia University, New York, New York, USA; ${ }^{2}$ Department of Epidemiology, Columbia University, New York, New York, USA; ${ }^{3}$ Division of Genetics, Wadsworth Center, New York State Department of Health, Albany, New York, USA; ${ }^{4}$ Department of Pediatrics, Weill Cornell Medical College, New York, New York, USA; ${ }^{5}$ Department of Neurology, Columbia University, New York, New York, USA; ${ }^{6}$ Columbia University School of Nursing, New York, New York, USA; ${ }^{7}$ Department of Medicine, Columbia University, New York, New York, USA. Correspondence: Wendy K. Chung (wkc15@columbia.edu)

The first two authors contributed equally to this work.

Submitted 10 April 2017; accepted 26 July 2017; advance online publication 12 October 2017. doi:10.1038/gim.2017.152
} 


\section{ORIGINAL RESEARCH ARTICLE}

mothers Monday through Friday at three hospitals in New York State (New York Presbyterian Morgan Stanley Children's Hospital, New York Presbyterian Allen Hospital, and New York Presbyterian Weill Cornell Medical Center) for 12 months beginning in January 2016. Study staff performed a brief chart review to determine study eligibility. Newborns with acute illness or congenital anomalies in the neonatal intensive care unit were excluded. Study staff provided a brief explanation of the study, a written brochure describing the study in English or Spanish, and the option to watch a fourminute video explaining the study (https://www.youtube.com/ watch?v = H_nIOEEZxh0). Parents provided electronic consent linked to a REDCap database. Routinely collected Guthrie cards were marked for SMA testing and sent to the Newborn Screening Program at the NYS Department of Health. The SMA test was provided at no cost to participants.

\section{SMN1 assay}

The SMN1 genotyping assay was designed and validated to detect carriers and homozygotes for the SMN1 exon 7 deletion. The deletion was detected by amplifying DNA extracted from one 3-mm dried blood spot ${ }^{7}$ using a multiplex TaqMan real-time quantitative polymerase chain reaction (qPCR) assay. ${ }^{8}$ Assays were run in triplicate on an Applied Biosystems 7900HT Real-Time PCR System or Quantstudio 12K Flex Real Time PCR System (Thermo Fisher Scientific, Waltham, MA). SMN1 exon 7 copy number was determined by calculating the relative quantity (RQ) using the $2^{-\Delta \Delta C t}$ method. ${ }^{9}$ Specimens not meeting quality control criteria, or those with a homozygous/heterozygous deletion, were rerun for confirmation using DNA extracted from a fresh dried blood spot punch. To rule out allelic dropout due to the presence of interfering sequence variants in the SMN1 TaqMan primer or probe binding sites, a fragment of exon 7 was sequenced in specimens with the SMN1 deletion. Assay details are included in the Supplementary Materials online.

\section{Notification of results}

Parents of one infant homozygous for the SMN1 deletion were notified by the study principal investigator, a medical geneticist, and asked to immediately come to the Columbia University Spinal Muscular Atrophy Clinical Research Center for evaluation and education about treatment options. A genetic counselor called all parents of heterozygous carriers of the SMN1 exon 7 deletion, and a letter documenting the results was mailed to families. Parents had the option of meeting with the genetic counselor and having genetic testing to determine if both parents were carriers for future family planning. Initially, parents of babies who screened negative for SMA were mailed a results letter, but this practice was terminated midway through the pilot to be consistent with return of results with routine newborn screening. Results for all babies were placed in the medical record with the rest of the newborn screening results.

\section{RESULTS}

\section{Validation study}

We evaluated the SMN1 qPCR assay using de-identified positive controls from the Pediatric Neuromuscular Clinical Research Network $(N=2)$ and Biogen, Idec $(N=45)$, and de-identified dried blood spots with unknown copy number $(N=4,028)$. There was a clear distinction between the average RQs of samples with 0,1 , and 2 or more copies of SMN1 exon 7, regardless of the SMN2 copy number. Based on these data, RQ bins were assigned for each SMN1 genotype, in which $\mathrm{RQ}=0.300-0.599$ were considered carriers, $\mathrm{RQ} \geq 0.800$ were considered screen negative, and $\mathrm{RQ}=0$ / undetermined were considered homozygous deletions. Two equivocal ranges, between 0 and 1 copy $(\mathrm{RQ}=0.001-0.299)$ and between 1 and 2 copies (0.600-0.799) were included. No samples fell into the equivocal range between 0 and 1 copy, and only 14 of the 4,028 samples $(0.35 \%)$ fell in the equivocal range between 1 and 2 copies. We suspected that suboptimal DNA extraction may have contributed to equivocal RQ values after they were resolved as normal $(N=5)$ or carriers $(N=9)$ with a second DNA extraction. It was decided that samples falling in the equivocal range would be repeated from a fresh dried blood spot punch.

There was $100 \%$ concordance between the copy number determined by our SMN1 qPCR assay and known genotypes of the 47 control samples. Individual replicates and average RQ values for sample replicates were consistent within and across runs. Of the 4,028 samples screened in the validation study, 51 (1.3\%) were SMA carriers, having one copy of SMN1 exon 7. None had a homozygous SMN1 deletion. Thirty-four $(0.84 \%)$ samples failed, which was attributed to poor DNA extraction.

The sequencing assay to check the sequence at the primer and probe binding sites was validated by testing several control samples with two copies of SMN1 and 40 samples with heterozygous deletions identified in the population study. Only the SMN1 gene, and not the SMN2 gene, was amplified and sequenced using primers designed to bind SMN1-specific nucleotides. No polymorphisms were identified in qPCR primer or probe binding regions of specimens with apparent heterozygous deletions.

\section{Newborn screening for SMA}

Between 14 January 2016 and 13 January 2017 a total of 3,826 babies born at the three New York Presbyterian Hospitals were screened for SMA. Across the three hospitals $93.03 \%$ of parents approached agreed to participate. The most common reasons for declining to participate were general distrust of the health-care system and prior bad experience with health care.

There were no technical issues with the SMN1 qPCR assay; $94.6 \%$ of results were released as screen negative without requiring retesting or confirmatory testing (Figure 1a). The first-pass assay failure/rejection rate was 3.0\%, which was attributed to suboptimal DNA quality and quantity; all were classified as screen negative or carriers upon retest 
(Figure 1b). An additional 33 specimens (0.86\%) initially tested in the equivocal range. Upon retesting using a fresh DNA sample, all but one resolved as screen negative $(N=30)$ or heterozygous deletions $(N=2)$. One specimen retested in the equivocal range. Upon sequencing, the specimen was found to carry a rare heterozygous sequence variant of uncertain significance (VOUS: c.842 G > C/p.Arg281Thr) that likely resulted in partial allelic dropout. Studies in vitro have shown that this variant does not affect the function of SMN1 gene product. ${ }^{10}$ This infant was classified as screen negative for this analysis, since the infant carried two copies of SMN1 exon 7 and the variant was detected as part of quality control measures. We do not know the clinical significance of the variant, and the result was reported to the family by the study genetic counselor as a VOUS.

SMA testing began the day after receipt by the lab, to allow for verification of specimens requisitioned for testing against the consent database. On day 2, DNA was extracted and the qPCR reactions were set up. Test results for all screen negatives were available on day 3 and available in the laboratory information management system on day 4 , which is within the release schedule for newborn screening results in NYS. Results from specimens requiring retest were typically available by day 5 . The median turnaround time for carriers, from day of receipt to final results, was six business days (range 3-11). Results for the VOUS carrier were available on day 7. Preliminary screen-positive results for the affected infant were communicated to the hospital on day 4 and confirmatory retesting was completed on day 5 .

Of the 3,826 babies screened, 3,766 specimens (98.43\%; 95\% confidence interval $(\mathrm{CI})=97.99-98.78 \%)$ had a normal screening result, $59(1.54 \% ; 1.20-1.98 \%)$ were identified as carriers, and $1(0.03 \% ; 0.00-0.15 \%)$ was homozygous for the SMN1 exon 7 deletion. The incidence of SMA in our population during the study period was 1 in 3,826; however, this estimate is imprecise due to the small sample size. The carrier frequency in this cohort was $1.5 \%(\mathrm{CI}=1.20-1.98 \%)$ or 1 in 65 , compared with prior estimates of 1 in 54 in the United States. ${ }^{2}$ Of the 3,826 babies screened, infant race and ethnicity was available for 1997 babies via chart abstraction. The carrier frequency was $1 / 44$ or $2.2 \%(\mathrm{CI}=1.29-3.72 \%$, $N=593$ ) for Caucasian, non-Hispanic, $1 / 126$ or $0.8 \%$ $(\mathrm{CI}=0.38-1.61 \%, N=893)$ for Hispanic, $1 / 49$ or $2.2 \%$ $(\mathrm{CI}=1.12-4.28 \%, N=364)$ for black, and $1 / 37$ or $2.7 \%$ $(\mathrm{CI}=1.06-6.79 \%, N=147)$ for Asian.

\section{SMA screen positive}

One patient was found to be homozygous for the SMN1 exon 7 deletion. She was evaluated at the Columbia University Spinal Muscular Atrophy Clinical Research Center at age 7 days. Genetic testing at an outside laboratory confirmed the homozygous SMN1 exon 7 deletion and the presence of two SMN2 copies. Her genotype correlated most closely with the SMA type 1 phenotype (Werdnig-Hoffman disease).

Upon initial evaluation at age 7 days, the baby had normal physical and neurological examinations. Tendon reflexes were
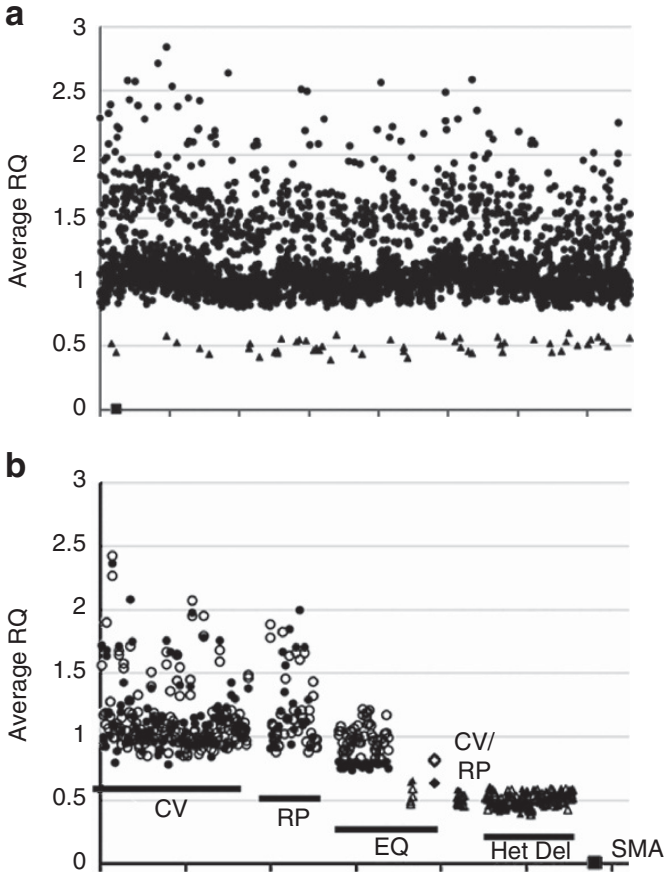

Figure 1 Average relative quantities for SMN1. (a) First-pass average relative quantities (RQs). Includes data from all subjects with an initial valid spinal muscular atrophy (SMA) screen falling into screen negative ( $R Q \geq 0.8 ; N=3,620$; circles), heterozygous deletion $(R Q=0.300$ $0.599 ; N=52$; triangles), or homozygous deletion $(R Q=0 ; N=1$; square) $\mathrm{RQ}$ ranges. Excludes screens that initially failed $\mathrm{QC}$ metrics (RNAseP Ct $\geq 35.0$ or $C V \geq 0.200 ; N=120$ ) or had average RQs in the equivocal range $(0.600-0.799 ; N=33)$. (b) Average RQs obtained from all samples requiring retesting. Average RQs for the initial (filled) and duplicate repeat tests (open) shown for each of 206 subjects requiring a repeat due to QC failure $(N=120 ; 3.1 \%)$, for those with an initial RQ in the equivocal range $(0.600-0.799 ; N=33 ; 0.9 \%)$, or for confirmation of heterozygous ( $N=52 ; 1.4 \%$ ) or homozygous deletions $(N=1 ; 0.03 \%)$. All but one sample (variant of uncertain significance carrier) initially in the equivocal range resolved as screen negative $(N=30)$ or carriers $(N=2)$ upon retesting. Results are shown according to final outcome (screen negative, circles; carriers, triangles; SMA, square; rare variant carrier, diamond). Initial replicates failing QC or falling in the equivocal range are included. CV, assay failure due to high coefficient of variation (CV $\geq 0.200$ ); CV/RP, high CV or RNAseP fail; $E Q$, average RQ falling in equivocal range (0.600-0.799); Het Del, heterozygous deletion; RP, assay replicate fail due to $R N A$ seP $C t \geq 35$; RQ, relative quantity, SMA, spinal muscular atrophy. A full color version of this figure is available at the Genetics in Medicine journal online.

present. At age 13 days the parents consented to enroll the baby in an open label study of nusinersen (ISIS 396443; now Spinraza) delivered intrathecally to infants with SMN1 disease-causing mutations who are genetically diagnosed with two or three SMN2 copies and are clinically presymptomatic (NURTURE) (ClinicalTrials.gov identifier: NCT02386553). Upon screening evaluation for the clinical trial, the baby was asymptomatic with a normal newborn clinical and neurological examination. Compound muscle action potential $(>1.5 \mathrm{mV})$ was normal, and she scored $55 / 64$ on the Children's Hospital of Philadelphia Infant Test of Neuromuscular Disorders motor function scale. ${ }^{11}$ 
At age 15 days, she received her first intrathecal injection of ISIS 396443. As of January 2017, at age 12 months, she had received six doses of nusinersen (ISIS 396443). She has met all of her developmental milestones within the typical temporal profile, sitting without support at 5 months, standing with assistance and crawling on hands and knees at 8 months, and cruising at 11 months. At the time of this report, she continues to be asymptomatic with a normal physical examination (https://youtu.be/fNz5ASV1x34 login: jk3906@cumc.columbia.edu password: genetics123). She remains in the 95th percentile for height and weight. She has not been hospitalized for any intercurrent illness and does not require any respiratory or nutritional support.

\section{DISCUSSION}

This pilot newborn screening study for SMA in NYS enrolled $93 \%$ of parents approached, successfully genotyped all enrolled newborns, and was successful in identifying one neonate with SMA. The frequency of SMN1 exon 7 deletion carriers we observed in this study population is approximately 1 in $65(1.5 \%)$, which is slightly lower than the carrier frequency of 1 in 54 reported in other studies. ${ }^{12-14}$ We report a carrier frequency of $1 / 44$ in the Caucasian non-Hispanic population, which is similar to other studies, and 1/49 in the black population, which is higher compared with other studies. $^{12}$ However, the carrier frequency was $1 / 126$ in Hispanics. A cross-sectional study of over 107,000 women in Taiwan from 2005 to 2009 using denaturing highperformance liquid chromatography and multiplex ligationdependent probe amplification yielded a SMA prevalence of 1 in 8,968 and carrier frequency of 1 in $48(2.10 \%) .{ }^{15}$ With our semiquantitative SMN1 assay we would not have identified carriers with the 2/0 SMN1 genotype with two copies of the normal SMN1 gene in cis, so there is a small chance to miss carriers (false negative), but not to miss homozygous SMN1 deletions.

In 2008, SMA was nominated to be added to the recommended uniform newborn screening panel. At the time, small studies had demonstrated that SMA could be accurately tested using DNA extracted from dried blood spots. ${ }^{16,17}$ However, the Advisory Committee on Heritable Disorders in Newborns and Children did not review the proposal, citing two primary deficiencies: a lack of prospective pilot studies demonstrating feasibility of SMA screening in a public health lab setting, and lack of effective treatment. Prior and colleagues ${ }^{18}$ completed a retrospective SMN1 screen in 40,000 de-identified dried blood spots obtained from the Ohio Department of Health. Taiwan began prospectively screening infants for SMA in a subset of their population after obtaining informed consent. A real-time PCR assay targeting a SMN1specific nucleotide in intron 7 (IVS7+100) was used. Beginning in 2014 through the end of 2015, more than 72,000 infants were screened, and five infants were identified with homozygous SMN1 mutations. The current NYS pilot study provides evidence that SMA can be reliably screened with high specificity and sensitivity, at high throughput, at reasonable cost, and within an acceptable turnaround time in a US public health lab setting.

More than $95 \%$ of SMA mutations are due to deletions in SMN1 exon $7,{ }^{19}$ therefore, we established a primary screen to detect the SMN1 exon 7 deletion. Approximately 5\% of SMN1 mutations are due to point mutations that would not have been detected by our assay. Newborns with the deletion were sequenced in the region surrounding the TaqMan primers to eliminate false positives due to polymorphisms in the primer/ probe binding sites. One of the 59 carriers had a heterozygous sequence variant (c. ${ }^{\star} 3+80 \mathrm{~T}>\mathrm{G}$; rs143838139) outside of the assay binding sites ( 80 nucleotides downstream of exon 7) that did not result in allelic dropout. The SMN1 qPCR assay is accurate and robust, with no identified technical issues. Other than the infant homozygous for the SMN1 deletion, we are not aware of any infants tested in this study who have been diagnosed with SMA (i.e., false negative screens) nor are we aware of any false positives.

In this study we were required to report carrier status because New York State Clinical Laboratory Standards of Practice (Genetic Testing Standard 12) states that any variant detected must be reported regardless of clinical implication. Other states may not have the same regulations and may decide not to report carrier status. Additional resources are required to report carrier results, and individual states will need to make decisions about capacity and value of providing carrier status. We provided genetic counseling by phone at the time of result disclosure, and few parents requested additional genetic counseling beyond the telephone conversation. The most immediate benefit of disclosing carrier status to parents is to inform family planning.

Most states also screen newborns for severe combined immunodeficiency (SCID) to identify newborns who could benefit from bone marrow transplant or other treatments. ${ }^{20}$ Labs with the capability to screen for SCID using real-time qPCR to detect T-cell receptor excision circles could use the existing molecular infrastructure to screen for SMA. Excluding labor, and assuming that DNA will already be extracted for SCID screening, the additional cost for qPCR reagents and consumables for the SMN1 exon 7 deletion assay is approximately $\$ 0.70$ per $10-\mu 1$ reaction.

Several changes to the pilot SMA screening algorithm would be implemented if SMA screening became mandated in NYS. Because the sample number is modest (mean $=15$ per day), DNA for SMA testing is extracted manually. For population-based screening, the use of DNA automatically extracted for SCID screening would decrease cost and labor. ${ }^{7}$ We also implemented conservative quality control criteria during the pilot phase. The assay is run in triplicate on a single DNA extract from all samples, permitting use of an average RQ to minimize repeat testing of samples falling into the equivocal RQ ranges, and a coefficient of variability that allows identification and retesting of samples with less precise RQ estimates. These metrics are relevant to distinguishing screen negatives from carriers. Our validation study and pilot testing have demonstrated that infants homozygous for the 
SMN1 deletion consistently screen positive, with an RQ of zero/undetermined for each replicate; conversely, infants with at least one copy of SMN1 exon 7 have RQs of at least 0.3.

To assess the impact of single rather than triplicate testing on SMA screening retest rates, we conducted a retrospective review of the 11,478 individual RQs for the 3,826 initial screens reported in this study. No replicates fell in the low equivocal range (0.001-0.299). However, 380 (3.3\%) fell into the high equivocal range (0.600-0.799), and would require retesting. Since the individual RQ values over all 531 initial and repeat tests among 59 infants with heterozygous deletions ranged from 0.33 to 0.712 , the upper limit of the equivocal range could be adjusted from 0.799 to 0.749 , which would reduce the number of equivocal test results from 3.3 to $1.1 \%$ and still correctly identify all heterozygous deletions. With elimination of triplicate testing, the overall retest rate for assay fails and equivocal/borderline results is predicted to decrease, from $3.86 \%$ using the current algorithm to $3.7 \%$. A concurrent adjustment in the cutoff could further reduce the retest rate to $1.5 \%$. These rates are similar to typical newborn screening retest rates. Furthermore, states considering implementation of SMA testing could opt out of reporting SMA carrier status. Without triplicate testing, the retest rate (calculated by including DNA extraction and assay fails) would fall to $0.4 \%$ if carrier status was not reported or was masked.

To reduce cost and labor, we validated a multiplex qPCR assay for simultaneous detection of $\mathrm{T}$-cell receptor excision circles and the SMN1 deletion. The cost to add SMN1 primer/ probe mix to the NYS SCID assay is approximately $\$ 0.10$ per $10-\mu l$ reaction. The Centers for Disease Control (CDC) has developed such an assay; however, several key differences exist. For example, the CDC assay cannot distinguish carriers from screen negatives (i.e., carrier status is masked), the assay targets SMN1 intron 7, and the test is carried out in situ, directly on a 2-mm dried blood spot punch without prior DNA extraction.

Since over $93 \%$ of parents agreed to add SMA to their baby's routine newborn screening, this suggests that there is a general acceptance of newborn screening for SMA, even before a proven treatment was available. This is similar to results reported by other newborn screening pilots for SMA, fragile X syndrome, and Pompe disease that were conducted in the United States and abroad. ${ }^{21-23}$ SMA preconception/ prenatal carrier screening is frequently offered to women, and some parents declined participating in this study because one or both parents previously had SMA carrier screening. With the availability of prenatal carrier screening and the realization of treatment for SMA during the one-year period of this study, we became aware of a couple from another state who elected to continue the pregnancy of a fetus prenatally diagnosed with SMA. We anticipate that the availability of treatment will influence reproductive decisions of other couples and, therefore, increase the importance of early identification of presymptomatic newborns who will realize the maximal clinical benefit from proactive treatment.
Telephone delivery of carrier results by a genetic counselor, followed up in writing, was straightforward and similar to our experience with returning carrier results for sickle cell disease on the routine newborn screening panel. Parents of babies who were carriers for SMA were offered an appointment with a genetic counselor. Generally, parents had no adverse reaction after finding out their child was a carrier for SMA. Most expressed concern, but after speaking with the genetic counselor understood "carrier" status versus "being affected." Ten families, including the parents of the infant carrying the VOUS, requested genetic counseling appointments, and two maintained the appointment. One couple had carrier screening for both parents to inform future family planning decisions.

The severity of SMA is related to SMN2 copy number, but $S M N 2$ copy number cannot perfectly predict phenotype. Most individuals with type 1 SMA have an SMN2 copy number of 2 , but $11 \%$ of type 2 SMA patients and $4 \%$ of type 3 SMA patients also have an SMN2 copy number of $2 .{ }^{24}$ Approximately $82 \%$ of SMA type 2 patients have an SMN2 copy number of 3 , but $20 \%$ of type 1 patients and $50 \%$ of type 3 patients also have three copies of SMN2. ${ }^{24}$ Greater than $45 \%$ of type 3 SMA patients have four copies of SMN2. ${ }^{24}$ Additionally, over $70 \%$ of patients with a SMN2 copy number of 2 have type 1 SMA. ${ }^{24}$ A variant in SMN2 exon 7, c. $859 \mathrm{G}>\mathrm{C}$, has also been reported to modify the SMA phenotype, and can cause allelic dropout for some SMN2 dosage assays. ${ }^{25}$ For quality assurance, we sequenced this region of the infant with SMA identified in this study. She does not carry this variant. Given the cost of nusinersen treatment and the lack of long-term outcome data about disease course with initiation of treatment in the newborn period, it is still unclear whether patients with more than four SMN2 copies should be treated as newborns and how frequently they should be treated. More data are necessary to inform that decision. We believe it is likely that such children would derive benefit, but it is unclear exactly what the optimal treatment protocol should be.

We describe the identification of a newborn expected to have type 1 SMA with a SMN2 copy number of 2 who began treatment with nusinersen within the first two weeks of life. To date, the baby at age 12 months remains asymptomatic and has met her motor and growth milestones appropriately. Given the feasibility of screening, the success of this pilot study, and the demonstrated efficacy and Food and Drug Administration approval of nusinersen in December 2016, we believe SMA should be considered for addition to the recommended uniform newborn screening panel.

\section{SUPPLEMENTARY MATERIAL}

Supplementary material is linked to the online version of the paper at http://www.nature.com/gim

\section{ACKNOWLEDGMENTS}

Funding was provided to Columbia University Medical Center by Biogen, Idec. Wadsworth Center contracted for laboratory testing from Columbia University Medical Center. We thank the patients 
and their families for participating, the Pediatric Neuromuscular Clinical Research Network and Biogen, Idec for contribution of DNA samples as controls. We thank the hospital staff, especially Jacqueline Gomez and Angela Pena; the newborn screening staff, especially Sandra Levin and Patrick Wilson; and Rosangel Cruz for her oversight of the NURTURE study at Columbia University.

\section{DISCLOSURE}

The authors declare no conflict of interest.

\section{REFERENCES}

1. Pearn J. Classification of spinal muscular atrophies. Lancet. 1980;1:919-922.

2. Kolb SJ, Kissel JT. Spinal muscular atrophy. Neurol Clin. 2015;33: 831-846.

3. Byers RK, Banker BQ. Infantile muscular atrophy. Arch Neurol. 1961;5: 140-164.

4. Muralidharan K, Wilson RB, Ogino S, Nagan N, Curtis C, Schrijver I. Population carrier screening for spinal muscular atrophy: a position statement of the Association for Molecular Pathology. J Mol Diagn. 2011;13:3-6.

5. Lefebvre S, Burglen L, Reboullet $\mathrm{S}$, et al. Identification and characterization of a spinal muscular atrophy-determining gene. Cell. 1995;80: $155-165$.

6. Cartegni L, Krainer AR. Disruption of an SF2/ASF-dependent exonic splicing enhancer in SMN2 causes spinal muscular atrophy in the absence of SMN1. Nat Genet. 2002;30:377-384.

7. Saavedra-Matiz CA, Isabelle JT, Biski CK, et al. Cost-effective and scalable DNA extraction method from dried blood spots. Clin Chem. 2013;59: 1045-1051.

8. Anhuf D, Eggermann T, Rudnik-Schoneborn S, Zerres K. Determination of SMN1 and SMN2 copy number using TaqMan technology. Hum Mutat. 2003:22:74-78.

9. Livak KJ, Schmittgen TD. Analysis of relative gene expression data using real-time quantitative PCR and the 2(-Delta Delta C(T)) Method. Methods. 2001;25:402-408.

10. Martin R, Gupta K, Ninan NS, Perry K, Van Duyne GD. The survival motor neuron protein forms soluble glycine zipper oligomers. Structure. 2012;20:1929-1939.

11. Glanzman AM, O'Hagen JM, McDermott MP, et al. Validation of the Expanded Hammersmith Functional Motor Scale in spinal muscular atrophy type II and III. J Child Neurol. 2011;26:1499-1507.
12. Sugarman $E A$, Nagan $N$, Zhu $H$, et al. Pan-ethnic carrier screening and prenatal diagnosis for spinal muscular atrophy: clinical laboratory analysis of $>72,400$ specimens. Eur J Hum Genet. 2012;20:27-32.

13. Hendrickson BC, Donohoe C, Akmaev VR, et al. Differences in SMN1 allele frequencies among ethnic groups within North America. J Med Genet. 2009;46:641-644.

14. Ogino S, Wilson RB. Genetic testing and risk assessment for spinal muscular atrophy (SMA). Hum Genet. 2002;111:477-500.

15. Su YN, Hung CC, Lin SY, et al. Carrier screening for spinal muscular atrophy (SMA) in 107,611 pregnant women during the period 20052009: a prospective population-based cohort study. PLoS One. 2011;6: e17067.

16. Pyatt RE, Prior TW. A feasibility study for the newborn screening of spinal muscular atrophy. Genet Med. 2006;8:428.

17. Majumdar R, Rehana Z, Al Jumah M, Fetaini N. Spinal muscular atrophy carrier screening by multiplex polymerase chain reaction using dried blood spot on filter paper. Ann Hum Genet. 2005;69(Pt 2): 216-21.

18. Prior TW, Snyder PJ, Rink BD et al. Newborn and carrier screening for spinal muscular atrophy. Am J Med Genet Part A. 2010;152A: 1608-1616.

19. Wirth B. An update of the mutation spectrum of the survival motor neuron gene (SMN1) in autosomal recessive spinal muscular atrophy (SMA). Hum Mutat. 2000;15:228-237.

20. Tagliaferri L, Kunz JB, Happich $M$, et al. Newborn screening for severe combined immunodeficiency using a novel and simplified method to measure T-cell excision circles (TREC). Clin Immunol. 2017; 175:51-55.

21. Famula J, Basuta K, Gane LW, Hagerman RJ, Tassone F. Identification of a male with fragile $X$ syndrome through newborn screening. Intractable Rare Dis Res. 2015;4:198-202.

22. Weinreich SS, Rigter $T$, van El CG, et al. Public support for neonatal screening for Pompe disease, a broad-phenotype condition. Orphanet $J$ Rare Dis. 2012;7:15.

23. Rothwell E, Anderson RA, Swoboda KJ, Stark L, Botkin JR. Public attitudes regarding a pilot study of newborn screening for spinal muscular atrophy. Am J Med Genet A. 2013;161A:679-686.

24. Feldkotter M, Schwarzer V, Wirth R, Wienker TF, Wirth B. Quantitative analyses of SMN1 and SMN2 based on real-time LightCycler PCR: fast and highly reliable carrier testing and prediction of severity of spinal muscular atrophy. Am J Hum Genet. 2002;70:358-368.

25. Prior TW, Krainer AR, Hua Y, et al. A positive modifier of spinal muscular atrophy in the SMN2 gene. Am J Hum Genet. 2009;85:408-413. 